\title{
openheart Physical Activity after Cardiac EventS (PACES): a group education programme with subsequent text message support designed to increase physical activity in individuals with diagnosed coronary heart disease: a randomised controlled trial
}

\author{
Louisa Y Herring (D) , ${ }^{1}$ Helen Dallosso (D) , ${ }^{1}$ Sally Schreder, ${ }^{1}$ Emily J Smith, ${ }^{2}$ \\ Ghazala Waheed, ${ }^{3}$ Laura J Gray, ${ }^{4}$ Kamlesh Khunti, ${ }^{3}$ Thomas Yates, ${ }^{3}$ \\ Patrick J Highton, ${ }^{3}$ Alex V Rowlands, ${ }^{3}$ Ian Hudson, ${ }^{5}$ Samuel Seidu (D) , ${ }^{3}$ \\ Melanie J Davies ${ }^{3}$
}

\begin{abstract}
- Additional material is published online only. To view please visit the journal online (http://dx.doi.org/10.1136/ openhrt-2020-001351).
\end{abstract}

To cite: Herring LY, Dallosso H, Schreder S, et al. Physical Activity after Cardiac EventS (PACES): a group education programme with subsequent text message support designed to increase physical activity in individuals with diagnosed coronary heart disease: a randomised controlled trial. Open Heart 2021;8:e001351. doi:10.1136/

openhrt-2020-001351

Received 29 May 2020 Revised 8 December 2020 Accepted 15 December 2020

Check for updates

(C) Author(s) (or their employer(s)) 2021. Re-use permitted under CC BY-NC. No commercial re-use. See rights and permissions. Published by BMJ.

For numbered affiliations see end of article.

Correspondence to Dr Louisa Y Herring; louisa. herring@uhl-tr.nhs.uk

\section{ABSTRACT}

Aim To assess the effectiveness of a low-cost pragmatic intervention (structured education and ongoing text message support) to increase daily physical activity in participants 12-48 months after a coronary heart disease cardiac event (myocardial infarction, angina or acute coronary syndrome) diagnosis.

Methods A single-centre randomised controlled trial of 291 adults randomised to a structured education programme $(n=145)$ or usual care $(n=146)$. The programme consisted of two 2.5 hour sessions delivered 2 weeks apart, followed by supplementary text message support. The GENEActiv accelerometer assessed the primary outcome at 12 months (change in overall physical activity (expressed in milli gravitational $(\mathrm{mg})$ units) from baseline). Secondary outcomes included anthropometric, physical function, cardiovascular, biochemical and patientreported outcome measures. Linear regression was used to compare outcome measures between groups on a modified intention-to-treat basis.

Results Participants' mean age was $66.5 \pm 9.7$ years, $84.5 \%$ males, $82.5 \%$ white British and $15.5 \%$ south Asian. At 12 months, there was no difference between the groups in terms of change in overall physical activity $(-0.23 \mathrm{mg}$ $(95 \% \mathrm{Cl}-1.22$ to 0.75$), \mathrm{p}=0.64)$ and the programme was well accepted ( $88 \%$ attendance). Exploratory analyses showed that average moderate to vigorous physical activity (MVPA) levels increased in individuals not meeting physical activity guidelines ( $\geq 150$ min per week) on enrolment compared with those who did, by 8 minutes per day $(8.04(95 \% \mathrm{Cl} 0.99$ to 15.10$), \mathrm{p}=0.03)$.

Conclusion The programme was well attended but showed no change in physical activity levels. Results show high baseline MVPA levels and suggest that Physical Activity after Cardiac EventS education may benefit cardiac patients not currently meeting activity guidelines. Trial registration number ISRCTN91163727.

\section{Key questions}

What is already known about this subject?

- Cardiac rehabilitation is an evidence-based complex intervention offered directly after a heart disease diagnosis, the intention being to improve modifiable risk factors such as cardiovascular risk, physical inactivity and psychological management to improve patients' quality of life and overall cardiovascular mortality. Cardiac rehabilitation uptake in the UK is only $50 \%$ and support is limited after discharge back to primary care, typically after 12 months post diagnosis.

What does this study add?

- To our knowledge, this is the first trial designed to assess the effectiveness of a structured selfmanagement group education programme 12-48 months after a cardiac event diagnosis to increase physical activity. The research shows no difference between groups for the primary outcome of total daily physical activity. However, the Physical Activity after Cardiac EventS (PACES) self-management education was very well accepted ( $88 \%$ attendance) in this multi-ethnic population and the programme was associated with increases in moderate to vigorous physical activity (MVPA) levels in cardiac patients not currently meeting the physical activity recommended guidelines ( $\geq 150$ mins MVPA per week). The PACES study recruited a high proportion of individuals who had not previously attended any form of cardiac rehabilitation.

\section{INTRODUCTION}

Undertaking regular physical activity is positively associated with coronary heart disease 


\section{Key questions}

How might this impact on clinical practice?

- Results suggest that structured self-management education programmes could be an option offered in parallel with cardiac rehabilitation; to capture those individuals not attending or implemented upon discharge back to normal care (12 months) to compliment current cardiac rehabilitation service provision.

(CHD) risk reduction and lower all-cause mortality, highlighting its importance in people with CHD. ${ }^{12}$ Cardiac rehabilitation is an evidence-based complex intervention offered to those following a cardiovascular event, targeting modifiable risk factors to improve patients' quality of life and overall cardiovascular mortality. ${ }^{3}$ Cardiac rehabilitation is clinically and cost-effective and should be offered to all those eligible to optimise patient outcomes. ${ }^{45}$ Cardiac rehabilitation uptake in the UK is $50 \%$ and support for patients is limited after discharge to primary care. ${ }^{4} \mathrm{~A}$ systematic review identified centrebased cardiac rehabilitation as insufficient for maintaining physical activity habits, ${ }^{6}$ however, research is limited. Cost-effective strategies to deliver interventions promoting self-management of health conditions are needed, as regular face-to-face contact with healthcare professionals is costly and arduous. ${ }^{7}$ Evidence highlights that more support is needed 1-year post cardiac event to complement existing services.

Research suggests the delivery of group-based structured education targeting lifestyle behaviour change is an effective and low-cost method of promoting selfmanagement of clinical conditions. ${ }^{8}$ This evidence, together with the increasing use of sophisticated mobile technologies and text messaging research, which have been shown to increase physical function, physical activity, medication adherence and improve cardiovascular risk factors, could be a beneficial combined support platform in CHD populations. ${ }^{9-11}$

The PACES study aimed to develop and assess the effectiveness of a low-cost pragmatic intervention (structured education and text message support) to increase daily physical activity in participants $12-48$ months post diagnosis of a cardiac event.

\section{METHODS}

\section{Study design}

A single-centre RCT with two parallel arms, the protocol has been previously described. ${ }^{7}$

\section{Participants}

Participants were recruited from the University Hospitals of Leicester National Health Service (NHS) Trust Cardiology Department and Phase Four community cardiac rehabilitation providers between March 2017 and March 2018 (recruitment target reached). Eligible participants were aged $\geq 18$ years, $12-48$ months after a CHD cardiac event diagnosis (myocardial infarction, angina, acute

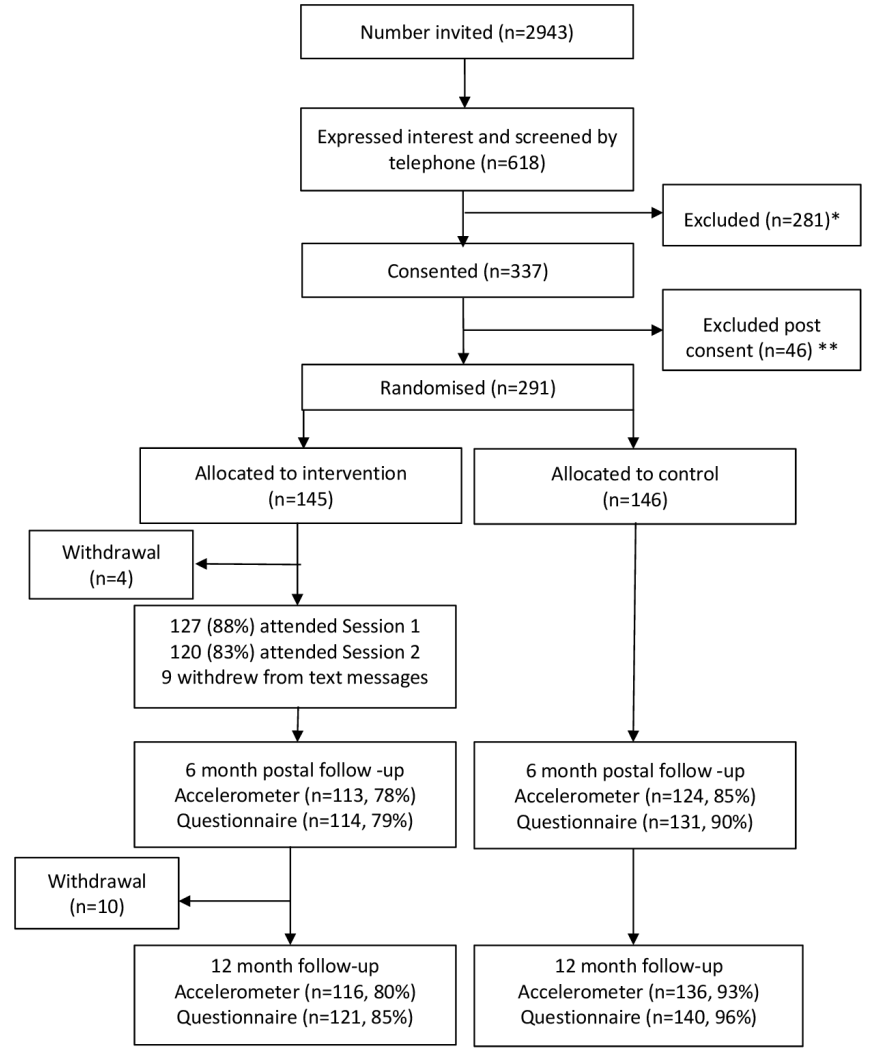

* Unable to attend education ( $n=38$ ), not between 12-48 months post diagnosis ( $n=31)$, poor ability to speak/ read English another life-threatening co-morbidity ( $n=10)$, unstable symptoms $(n=10)$, musculoskeletal limitations $(n=9)$, participation in another trial $(n=7)$, housebound or immobile $(n=7)$, primary diagnosis heart failure $(n=4)$, no mobile phone access $(n=4)$,
other $(n=149) ; 25 \%$ reached recruitment target, $20 \%$ health problems, $18 \%$ changed mind, $11 \%$ limited walking ability, No CHD)

**Blood pressure $\geq 160 / 100 \mathrm{mmHg}(n=24)$, did not achieve $120 \mathrm{~m}$ in ISWT ( $n=11)$, no history of CHD or cardiac event $(n=3)$ unable to speak/ understand English ( $n=1)$, not between $12-48 m$ post cardiac event $(n=3)$, under investigation $(n=3)$, very low pulse rate $(n=1)$

Figure 1 PACES study flow diagram. CHD, coronary heart disease; ISWT, incremental shuttle walk test; PACES, Physical Activity after Cardiac EventS.

coronary syndrome (ACS)), had mobile phone access, willing to allow their general practitioner (GP) notification of study participation and medical records access (study purposes), willing to attend education sessions and able to speak and read English. Potential participants were screened both via telephone and in the baseline assessment to ensure the exclusion criteria was not met (diagnosis of heart failure where the underlying primary cause was not myocardial disease as a result of atherosclerosis, any unstable symptoms (chest pain or breathlessness at rest; unstable stage II hypertension $(160 / 100 \mathrm{~mm} \mathrm{Hg})$, not on necessary medications), poor exercise capacity $(<120 \mathrm{~m}$ on incremental shuttle walk test (ISWT) $),{ }^{12}$ participating in another study, a severe life threatening comorbidity, housebound/immobile or musculoskeletal limitations that limit physical activity). If deemed eligible, informed consent was obtained (figure 1).

\section{Randomisation and masking}

On successful completion of visit 1 (baseline), participants were randomly allocated, using a 1:1 block design, stratified by gender (men; women) and ethnicity (White European; other), to receive either standard management in primary care (control) or to attend group-based 
structured education with text message support (intervention). An independent statistician developed the randomisation schedule and an independent researcher undertook randomisation allocation. Participants were informed of their randomisation allocation after attending visit 1 . Research nurses undertaking data collection and the staff processing accelerometer data were unaware of the randomisation outcome.

\section{Study groups}

Control group: Participants were provided with general health advice (British Heart Foundation (BHF) information leaflet 'Put Your Heart into Walking'13 after visit 1, and returned to their GP's standard care.

Intervention group: Participants received the BHF leaflet and were invited to attend the PACES education programme which comprised two group-based structured education sessions, developed via extensive patient and public involvement (see protocol paper for details). ${ }^{7}$ The sessions were two and a half hours in duration and delivered approximately 2 weeks apart by two trained facilitators. A facilitative style of delivery encouraged participation through reflective questioning and problem-solving activities promoted engagement and built self-efficacy. The programme content was underpinned by an integrated theoretical framework concentrating on the adoption and maintenance of an active lifestyle by increasing daily walking.

Education session attendees subsequently received physical activity related motivational reinforcement text messages. Participants received 82 non-interactive messages at different weekly frequencies. The text message support used was a validated package of specifically designed messages effective in the prevention of recurrent cardiovascular events. ${ }^{914}$

\section{Outcome measures}

Measurements were obtained at baseline, 6 months (postal assessment of objectively measured physical behaviours and questionnaires only) and 12 months. Demographic and medical history data were recorded. For details of the measurement of secondary outcomes, see protocol. ${ }^{7}$

\section{Objectively measured physical behaviours}

Change in overall daily physical activity (average acceleration measured in $\mathrm{m} g$ ) from baseline to 12 months was the primary outcome, collected at all three time points using a wrist worn triaxial accelerometer (GENEActiv model 1.1, ActivInsights, Cambridgeshire, UK). Participants were given the GENEActiv accelerometer when they attended the data collection session and asked to wear it on their non-dominant wrist for the following eight consecutive days (24 hours) after which they returned it to the research team in a prepaid envelope. Participants completed a wear-time diary and sleep log. Configuration of the GENEActiv ensured data were collected at $100 \mathrm{~Hz}$.
Physical function measurement

The ISWT was used as a screening tool and outcome measure. It is regularly used in cardiac rehabilitation and reflects walking ability, an important measure of daily living. ${ }^{12}$

\section{Pathology Samples}

Venous blood samples were obtained (full lipid profile and glycated haemoglobin (HbAlc)) and analysed in University Hospitals of Leicester NHS Trust accredited laboratories.

\section{Questionnaire Data}

The following validated questionnaires were administered at all three assessments: Recent Physical Activity Questionnaire, which explores day-to-day physical activity levels in the past 4 weeks ${ }^{15}$; Hospital Anxiety and Depression Scale, administered to measure anxiety and depression symptoms ${ }^{16}$; EuroQoL-5 Dimension-5 Level health status measurement tool, which assesses health-related quality of life ${ }^{17}$; Jenkins self-efficacy scale, which measures ability to continue exercising when faced with nine different barriers to exercise ${ }^{18}$; MacNew Heart Disease health-related quality of life questionnaire which measures how CHD affects emotional, physical and social functioning along with daily activity. ${ }^{19}$

\section{Sample size}

In order to detect a minimum clinically significant difference in overall daily physical activity (average acceleration) of $2.1 \mathrm{mg}$ in change from baseline to 12 months, (estimated to be equivalent to an increase of approximately $30 \mathrm{~min}$ of light walking at $4 \mathrm{~km}$ /hour), assuming a SD of $5.3 \mathrm{mg},{ }^{20}$ a power of $80 \%$ and significance level of 5\%, the sample size required was 202 participants. To allow for $20 \%$ loss to follow-up and $10 \%$ non-compliance of the GENEActiv, 290 participants were required.

\section{Data analysis}

A statistical analysis plan was agreed prior to data analysis. Baseline characteristics were summarised by intervention arm. Continuous variables were expressed as mean values (SD), or median values (with lower and upper quartiles) where appropriate. Categorical variables were expressed as number (percentage). Data were checked for parametric assumptions.

\section{Primary outcome data processing and analysis}

A complete case population (ie, those with complete data for the primary outcome and stratification factors) was used for the primary analysis, retaining randomisation (ie, a modified intention-to-treat analysis).

The accelerometer data were downloaded and processed ahead of analysis with R-package GGIR V.1.9 (http://cran.r-project.org). ${ }^{21} \quad$ Autocalibration was undertaken using local gravity as a reference, direction of sustained abnormally high values, calculation of the average resultant vector magnitude, corrected for gravity and expressed as Euclidean Norm Minus One in 
$\mathrm{m} g$ averaged over $5 \mathrm{~s}$ epochs. Files were excluded from analyses if post calibration error was greater than $0.01 \mathrm{~g}$ or fewer than 4 days of 16 hours of monitor wear time. Non-wear was estimated based on the SD and value range of each axis, calculated for $60 \mathrm{~min}$ windows with $15 \mathrm{~min}$ moving increments. If for at least two of the three axes the SD was less than $13 \mathrm{~m} g$ or the value range was less than 50 $\mathrm{m} g$ the time window was classified as non-wear. Outcomes included overall physical activity, moderate to vigorous physical activity (MVPA)) accrued in 1 min bouts, intensity of the most active continuous $30 \mathrm{~min}$ per day $(\mathrm{m} g)$, daily MVPA (>100 mg) accrued in 1 min bouts, ${ }^{22}$ sedentary time ( $<40 \mathrm{mg}$, excluding sleep), and sleep duration. Estimation of time spent sleeping was guided by the sleep $\log$ and excluded waking periods in the night.

Primary outcome data were compared using linear regression with a binary indicator for randomisation group as the explanatory variable, terms for stratification factors (gender and ethnicity) as confounders and adjustment for change from baseline in accelerometer wear time and baseline overall daily physical activity. This included participants who had worn the accelerometer for at least four valid days. A sensitivity analysis was carried out on a full intention-to-treat (using multiple imputation) and per-protocol basis (defined as participants who attended at least one PACES education session, with those who attended less excluded from the intervention arm). The multiple imputation model included overall physical activity at 12 months, intervention group, stratification factors (sex and ethnicity) and overall physical activity at baseline. Rubin's formula was used to combine the parameter estimates and standard errors from 100 imputations into a single set of results. ${ }^{23}$ Interactions were fitted in the primary analysis between intervention arm and age, sex, ethnicity and ISWT distance to assess whether the treatment effect differed by these groups. Secondary outcomes were analysed using similar methods, with an appropriate model selected dependent on the outcome distribution.

Further exploratory analyses were undertaken to describe the results observed. Intervention participants were stratified into those not meeting current MVPA guidelines $(<150$ min per week) on recruitment versus those who were $(\geq 150$ min per week). These analyses were not prespecified and should be viewed as hypothesis generating. Changes in physical activity measures were compared between these groups using linear regression adjusted for average wear time.

Statistical significance for main effects were assessed at the $5 \%$ level with $95 \%$ CIs, interactions were assessed at $10 \%$ level, all $\mathrm{p}$ values are two sided.

\section{RESULTS}

\section{Participant characteristics}

Figure 1 shows 2943 patients invited with 618 (21\%) expressing an interest and receiving telephone eligibility screening. Of these, 337 patients gave consent and undertook screening in clinic, with 291 participants identified as eligible and randomised. Eighty-eight per cent of intervention participants $(\mathrm{n}=127)$ attended a minimum of one education session with $83 \% \quad(n=120)$ attending both. Withdrawal from the text message support package was low $(n=9)$.

The mean duration since the participant's cardiac event was $29.0 \pm 9.3$ months. Mean age was $66.5 \pm 9.7$ years, $84.5 \%$ were males, $77 \%$ previously attended cardiac rehabilitation, $82.5 \%$ white European and $15.5 \%$ south Asian ethnicity. At baseline mean body mass index (BMI) and body weight were $29.1 \pm 4.3 \mathrm{~kg} \cdot \mathrm{m}^{2}$ and $83.6 \pm 15.1 \mathrm{~kg}$, respectively (table 1). Overall the groups were comparable for all key variables.

\section{Primary outcome}

Complete case analysis, found no significant difference between the control and intervention groups for change in overall daily physical activity $(\mathrm{mg})$ at 12 months (diff $-0.23 ; 95 \% \mathrm{CI}-1.22$ to $0.75 ; \mathrm{p}=0.642$ ). This was confirmed in the sensitivity analyses for both intention-to-treat and per protocol (table 2). Subgroup analysis showed no significant differences between the control and intervention groups in the primary outcome for age, sex, ethnicity and ISWT distance (online supplemental figure 1).

\section{Secondary outcomes}

Physical activity measures from accelerometer data, selfreported physical activity, clinical measures and patientreported outcomes showed no difference between groups (online supplemental tables 1-4) apart from a significant reduction in self-reported sedentary time $(\leq 1.5$ metabolic equivalents (METs)) at 6 months and 12 months (expressed as hours per day) favouring the intervention group (online supplemental table 3 ).

\section{Exploratory analysis}

Exploratory analyses investigated change from baseline in physical activity measures in the intervention group participants not meeting MVPA guidelines $(<150 \mathrm{~min}$ weekly) on recruitment versus those who did ( $\geq 150 \mathrm{~min}$ weekly). A statistically significant difference in MVPA bouts was seen, favouring those not meeting the guidelines by 8.0 min per day ( 56.3 min weekly) compared with those meeting the guidelines (table 3 ).

\section{Adverse events}

Seven serious adverse events were reported, none of which related directly to the intervention. Two adverse events were reported as possibly related (online supplemental table 5).

\section{DISCUSSION}

The PACES intervention programme was not associated with an improvement in overall PA. However, the programme's uptake and completion rates were very high, with $23 \%$ of those attending not having previously received any form of cardiac rehabilitation post cardiac 


\section{Health care delivery, economics and global health care}

Table 1 Baseline characteristics by randomised groups; usual practice (control) verses structured education (intervention)

\begin{tabular}{|c|c|c|c|}
\hline Characteristics & $\begin{array}{l}\text { Control } \\
n=146\end{array}$ & $\begin{array}{l}\text { Intervention } \\
\mathrm{n}=145\end{array}$ & $\begin{array}{l}\text { Total } \\
\mathrm{n}=291\end{array}$ \\
\hline \multicolumn{4}{|l|}{ Demographic measurements } \\
\hline Age (years) & $66.6(10.2)$ & $66.48(9.2)$ & $66.5(9.7)$ \\
\hline \multicolumn{4}{|l|}{ Gender } \\
\hline Male, n (\%) & $124(85)$ & $122(84)$ & $246(84)$ \\
\hline \multicolumn{4}{|l|}{ Ethnicity } \\
\hline White British, n (\%) & $121(83)$ & 119 (82) & 240 (82) \\
\hline South Asian, $\mathrm{n}(\%)$ & $22(15)$ & $23(16)$ & $45(16)$ \\
\hline Others, n (\%) & $3(2)$ & $3(2)$ & $6(2)$ \\
\hline \multicolumn{4}{|l|}{ Smoking status } \\
\hline Current smoker, n (\%) & $5(3)$ & $8(5)$ & $13(4)$ \\
\hline Former smoker, $\mathrm{n}(\%)$ & $82(56)$ & $64(44)$ & $146(50)$ \\
\hline Never smoked, n (\%) & $56(38)$ & $70(48)$ & $126(43)$ \\
\hline Alcohol intake (yes), n (\%) & $93(64)$ & $87(61)$ & $180(63)$ \\
\hline \multicolumn{4}{|l|}{ Employment status } \\
\hline Employed, n (\%) & $51(35)$ & $53(37)$ & $104(36)$ \\
\hline Unemployed, n (\%) & $5(3)$ & $3(2)$ & $8(3)$ \\
\hline Retired, $\mathrm{n}(\%)$ & $90(62)$ & $88(61)$ & $178(61)$ \\
\hline \multicolumn{4}{|l|}{ Anthropometric measurements } \\
\hline Body weight (kg) & $83.5(14.0)$ & $83.8(16.1)$ & $83.6(15.1)$ \\
\hline Body mass Index $\left(\mathrm{kg} / \mathrm{m}^{2}\right)$ & $28.8(4.0)$ & $29.3(4.7)$ & $29.1(4.3)$ \\
\hline Waist (cm) & $100.6(11.5)$ & $101.5(11.8)$ & $101.1(11.6)$ \\
\hline Hip (cm) & $104.3(8.5)$ & $104.8(8.3)$ & $104.6(8.4)$ \\
\hline Waist to hip ratio $(\mathrm{cm})$ & $0.96(0.1)$ & $0.96(0.1)$ & $0.96(0.1)$ \\
\hline \multicolumn{4}{|l|}{ Cardiovascular measurements } \\
\hline Systolic blood pressure $(\mathrm{mm} \mathrm{Hg})$ & $125(15.6)$ & $126(16.2)$ & $126(15.8)$ \\
\hline Diastolic blood pressure (mm Hg) & $71(10.2)$ & $72(11.3)$ & $72(10.7)$ \\
\hline Heart rate (bpm) & $61(9.7)$ & $60(8.6)$ & $60(9.1)$ \\
\hline \multicolumn{4}{|l|}{ Biomedical measurements } \\
\hline Total cholesterol (mmol/L) & $4.0(0.9)$ & $4.0(0.9)$ & $4.0(0.9)$ \\
\hline Triglycerides (mmol/L)) & $1.6(0.9)$ & $1.6(0.8)$ & $1.6(0.8)$ \\
\hline $\mathrm{HDL}-\mathrm{C}(\mathrm{mmol} / \mathrm{L})$ & $1.3(0.3)$ & $1.3(0.3)$ & $1.3(0.3)$ \\
\hline LDL-C (mmol/L) & $2.0(0.8)$ & $2.0(0.7)$ & $2.0(0.7)$ \\
\hline Total cholesterol-HDL ratio (mmol/L) & $3.3(0.9)$ & $3.2(0.8)$ & $3.2(0.9)$ \\
\hline HbA1c (\%) & $6.0(0.9)$ & $6.1(0.9)$ & $6.0(0.9)$ \\
\hline $\mathrm{HbA1c}(\mathrm{mmol} / \mathrm{mol})$ & $42(9.4)$ & $43(9.8)$ & $43(9.6)$ \\
\hline \multicolumn{4}{|l|}{ Accelerometer variables } \\
\hline Daily average acceleration $(\mathrm{mg})$ & $24.0(8.0)$ & $23.3(6.8)$ & $23.6(7.4)$ \\
\hline Av acceleration for most active $30 \mathrm{mins}(\mathrm{mg})$ & $77.7(62.0)$ & $70.0(28.3)$ & $73.8(48.1)$ \\
\hline MVPA 1 min bout (mins) & $29.7(23.9)$ & $29.1(26.3)$ & $29.4(25.1)$ \\
\hline Sedentary/inactive timer per day (mins) & $760(103)$ & $764(101)$ & $762(102)$ \\
\hline Sleep duration per night (mins) & $374(71)$ & $373(72)$ & $374(72)$ \\
\hline No of valid activity days & $6.9(0.5)$ & $6.9(0.4)$ & $6.9(0.5)$ \\
\hline \multicolumn{4}{|l|}{ Medical history } \\
\hline T2DM, n (\%) & $26(18)$ & $26(18)$ & $52(18)$ \\
\hline
\end{tabular}




\begin{tabular}{lccc}
\hline Table 1 Continued & & & \\
\hline Characteristics & $\begin{array}{l}\text { Control } \\
\mathbf{n}=\mathbf{1 4 6}\end{array}$ & $\begin{array}{l}\text { Intervention } \\
\mathbf{n}=\mathbf{1 4 5}\end{array}$ & $\begin{array}{l}\text { Total } \\
\mathbf{n}=\mathbf{2 9 1}\end{array}$ \\
\hline Hypertension, $\mathrm{n}(\%)$ & $48(33)$ & $66(46)$ & $114(40)$ \\
Myocardial Infarction, $\mathrm{n}(\%)$ & $89(61)$ & $92(63)$ & $181(62)$ \\
Incremental shuttle walk test & & $330(133)$ & $327(141)$ \\
ISWT distance $(\mathrm{m})$ & $324(149)$ & & \\
\hline
\end{tabular}

Missing data: 6 smoking status; 4 alcohol; 1 employment status; 1 waist-to-hip ratio; 4 total cholesterol; 4 HDL cholesterol; 5 LDL cholesterol; $8 \mathrm{HbA1c} 8$ overall accelerometer variables; 0 all other variables.

There were no statistically significant differences between the two arms for all characteristics.

Data given as mean (SD) unless stated.

HDL-C, high-density lipoprotein cholesterol; ISWT, incremental shuttle walk test; LDL-C, low-density lipoprotein cholesterol ; MVPA, moderate to vigorous intensity physical activity; T2DM, type 2 diabetes mellitus.

event. On average $54 \%$ of participants were surpassing weekly physical activity guidelines at baseline by $\sim 56$ MVPA minutes weekly (37\% greater). Exploratory analysis suggests individuals not meeting the physical activity guidelines at baseline, displayed a superior response to the PACES programme, by increasing MVPA.

Overall mean activity levels displayed a small increase at 6 months and a slight reduction at 12 months, although non-significant. Physical activity guidelines recommend adults should perform 150 MVPA minutes weekly. ${ }^{4}$ At baseline participants performed on average 206 MVPA minutes, suggesting that the capacity for benefit was limited in this group and likely explaining the lack of intervention-induced improvements. A recent systematic review suggests that a higher proportion of individuals who have attended cardiac rehabilitation are classed as physically active when compared with non-attendees ${ }^{24}$; this potentially explains why the current study elicits high MVPA levels. Seventy-seven percent of the PACES participants had previously attended cardiac rehabilitation, which is $27 \%$ and $14 \%$ higher than the average attendance nationally (50\%) and locally $(63 \%) .{ }^{424}$ Capturing a high proportion of individuals who had attended cardiac rehabilitation may have contributed to the minimal change in physical activity observed due to high baseline activity levels. Furthermore, as participation bias is common in physical activity research ${ }^{25}$ some physical activity or health-related selection bias may have been introduced, resulting in a more active, healthy and medically well-managed sample with respect to the typical population. Recruiting an already active group who have adopted the necessary lifestyle changes recommended to self-manage their cardiac condition limits the potential lifestyle changes promoted by the PACES education programme. This notion is supported by the relatively low low-density lipoprotein cholesterol (LDL-C) values, number of non-smokers and average age of the recruited sample. These factors, combined with the duration since cardiac event (12-48 months) and the high previous attendance at cardiac rehabilitation (77\%) may have meant that the sample were largely too active and healthy to achieve any significant intervention-induced improvement in physical activity levels.

In further support of this theory, individuals in the intervention group not meeting physical activity guidelines at baseline displayed an improvement in MVPA

Table 2 Changes in overall physical activity for 4 valid days of wearing accelerometer at 12- month follow-up between participants randomised to usual practice (control) or to structured education (intervention)

\begin{tabular}{|c|c|c|c|c|c|c|}
\hline & \multicolumn{2}{|c|}{ No of participants } & \multicolumn{2}{|c|}{$\begin{array}{l}\text { Mean change from } \\
\text { baseline }\end{array}$} & \multicolumn{2}{|c|}{$\begin{array}{l}\text { Adjusted difference at follow- } \\
\text { up* }\end{array}$} \\
\hline & $\begin{array}{l}\text { Control } \\
(n=146)\end{array}$ & $\begin{array}{l}\text { Intervention } \\
(n=145)\end{array}$ & Control & Intervention & Coefficient $(95 \% \mathrm{Cl})$ & $P$ value \\
\hline \multicolumn{7}{|l|}{ Complete case analysis $\dagger$} \\
\hline Overall physical activity (mg/day) & 130 & 115 & -0.28 & -0.38 & $-0.23(-1.22$ to 0.75$)$ & 0.642 \\
\hline \multicolumn{7}{|l|}{ Intention to treat analysisł } \\
\hline Overall physical activity ( $\mathrm{m} g /$ day) & 146 & 145 & -0.28 & -0.41 & $-0.25(-1.23$ to 0.74$)$ & 0.622 \\
\hline \multicolumn{7}{|l|}{ Per protocol analysis§ } \\
\hline Overall physical activity (mg/day) & 130 & 103 & -0.28 & -0.29 & -0.16 (-1.18 to 0.86$)$ & 0.759 \\
\hline
\end{tabular}

*Adjusted for stratification factors: sex and ethnicity; change from baseline in accelerometer wear time and baseline value of outcome.

†Participants with missing outcome data or missing variables required for the model adjustment were excluded.

$\ddagger$ Missing data imputed using multiple imputation.

§Participants who did not engage with at least one group session of the programme have been excluded from the intervention arm. 
Table 3 Exploratory analysis to investigate the change in physical activity stratified by baseline MVPA $<150$ min/week (low activity) versus MVPA $\geq 150 \mathrm{~min} /$ week (high activity) in the intervention group participants

\begin{tabular}{|c|c|c|c|c|}
\hline & Mean change from $b$ & line & $\begin{array}{l}\text { Adjusted difference for mean } \\
\text { change from baseline* }\end{array}$ & \\
\hline & $\begin{array}{l}\text { MVPA } \geq 150 \mathrm{mins} / \mathrm{wk} \\
(\mathrm{n}=75)\end{array}$ & $\begin{array}{l}\text { MVPA }<150 \text { mins } / \\
\text { wk }(n=68)\end{array}$ & Coefficient $(95 \% \mathrm{Cl})$ & P value \\
\hline Overall physical activity (mg/day) & -0.74 & 0.07 & $0.83(-0.68$ to 2.34$)$ & 0.279 \\
\hline Mean at baseline & 27.23 & 18.88 & & \\
\hline Mean at 12 months & 25.91 & 18.9 & & \\
\hline Intensity of the most active 30 mins (mg/day) & -5.52 & 2.42 & $7.91(-1.53$ to 17.36$)$ & 0.1 \\
\hline Mean at baseline & 86.28 & 51.93 & & \\
\hline Mean at 12 months & 79.75 & 54.76 & & \\
\hline MVPA (1 min bouts) (mins/day) & -5.6 & 2.24 & $8.04(0.99$ to 15.10$)$ & 0.026 \\
\hline Mean at baseline & 46.67 & 9.63 & & \\
\hline Mean at 12 months & 40.51 & 11.72 & & \\
\hline Sedentary/inactive time per day ( $\mathrm{min}$ ) & 1.82 & 13.02 & $11.77(-21.44$ to 44.98$)$ & 0.484 \\
\hline Mean at baseline & 729.65 & 802.57 & & \\
\hline Mean at 12 months & 734.55 & 802.44 & & \\
\hline Sleep duration per night (mins) & -0.9 & -13.15 & $-14.03(-34.48$ to 6.42$)$ & 0.177 \\
\hline Mean at baseline & 370.17 & 376.4 & & \\
\hline Mean at 12 months & 370.66 & 371.63 & & \\
\hline
\end{tabular}

${ }^{\star}$ Adjusted for change from baseline in accelerometer wear time. Reference group: MVPA $\geq 150$.

MVPA, Moderate to vigorous physical activity based on the $1 \mathrm{~min}$ bout baseline data.

compared with those meeting the guidelines in the non-prespecified exploratory analysis (table 3). MVPA improved in this group by 2.24 min per day by 12 months ( 16 MVPA minutes weekly), a 23\% increase from baseline. A minimum clinically important difference in MVPA in a clinical population has been reported between 8 and 26 min weekly. ${ }^{26}$ This increase with the reduction in the group achieving the guidelines suggests the PACES programme may benefit a less active cardiac population. For instance, a previous study that investigated a similar intervention (though also including four supervised exercise sessions) in an older cohort of patients (76 years) with reduced physical performance only 1-month post hospitalisation for ACS, found a significant improvement in measures of physical function. ${ }^{27}$ This highlights the importance of identifying those at greatest need (ie, those with reduced physical activity or function) prior to undertaking physical activity interventions. This previous study also found improved quality of life scores in the intervention group, while no change in quality of life was observed here. As cardiac rehabilitation tends to improve quality of life, which is in part mediated by increased physical activity levels,${ }^{28}$ it is possible that, as with physical activity, baseline quality of life levels were too high in the group studied here, thus limiting the capacity for benefit.

Several participants $(23 \%)$ had not previously attended cardiac rehabilitation; potentially indicating that structured self-management education in these individuals is preferred to cardiac rehabilitation pathways currently offered (supervised exercise/online support). ${ }^{4}$
Alternatively, this may suggest that some patients are not ready to make physical activity lifestyle modifications directly after their cardiac event, needing the additional support 12-48 months later. At this point, patients have been discharged back to usual care with limited cardiac support available. This could suggest that self-management education, similar to the PACES programme, could complement current cardiac rehabilitation services capturing non-attendees 12 months post cardiac event diagnosis. This finding and the indication that PACES education improves MVPA levels in participants not achieving physical activity guidelines could support emerging research suggesting a more tailored cardiac care approach is needed ${ }^{29}$ Stratification of individuals to the most relevant services could create a more person-centred care approach to their disease management. ${ }^{29}$

The characteristics of PACES study participants and cardiac rehabilitation attendees are comparable (national audit of cardiac rehabilitation (NACR) report (a large representative UK cardiac rehabilitation database)). PACES participants were on average 66 years old, with a $29 \mathrm{~kg} / \mathrm{m}^{2}$ BMI, $84.5 \%$ were male, $82.5 \%$ white British and $15.5 \%$ south Asian ethnicity. ${ }^{4}$ Notable differences include a higher proportion of South Asian individuals ( $10.5 \%$ greater) and males ( $14.2 \%$ greater) recruited to PACES than reported by NACR, although ethnicity did not impact on the study findings. ${ }^{4}$ Cardiac rehabilitation's reported dropout rate is $20 \%-30 \%(\sim 24 \%)^{4}$; only $12 \%$ did not complete the PACES education programme, 
potentially attributable to a shorter programme. Structured self-management education is common practice in the prevention and management of chronic diseases; previous research in a diabetes population reports $8 \%$ uptake and $19.5 \%$ attendance at such programmes. ${ }^{30} 31$ PACES displayed a $21 \%$ positive response rate to study participation, with $88 \%$ of intervention participants completing the education programme and 90\% (261) of the total sample completing the 12-month follow-up. Completion rates for the cardiac rehabilitation core component is $76 \%$, with $63 \%$ attending both the core component and final assessment. ${ }^{4}$ These attendance figures are significantly greater than what is typically reported. ${ }^{4031}$ This high uptake, high retention and positive feedback could indicate that structured selfmanagement with text messaging support is well received in a cardiac population and considered important 12-48 months post surgery when specialist cardiac support is no longer readily available.

Study strengths include objectively measured physical activity, a multi-ethnic population, high retention and follow-up rates. Since the protocol conception and close of recruitment, the research field has evolved and data now suggest that smaller levels of physical activity are clinically meaningful. A change in 500 steps per day is consistent with a change in daily average acceleration of approximately $0.9 \mathrm{mg}$ which is now deemed clinically important; the study would, therefore, be underpowered to detect such small improvements. ${ }^{32}$ Limitations may comprise the physical activity ISWT exclusion criterion which meant potentially excluding the most inactive individuals (ISWT included for safety purposes) and the single-centre study design. Future research should assess structured self-management education programmes for increasing physical activity in an inactive population, screening out individuals already meeting physical activity guidelines and target physical activity maintenance for those already active. Similarly, translating this approach to more recently after a cardiac event may increase the likelihood of efficacy by supporting patients to make lifestyle changes during the rehabilitation period, or by capturing those who do not attend cardiac rehabilitation ( $50 \%$ nationally).

In conclusion, the PACES study recruited an already well managed clinical group whose mean baseline physical activity level surpassed the national physical activity guidelines, possibly explaining why attending the programme did not appear to benefit physical activity. ${ }^{22}$ Results suggest that PACES education may be more beneficial for a less active cardiac population. This, with the high uptake, retention rates and recruitment of a large proportion of individuals who had not previously taken part in cardiac rehabilitation, suggests support is needed 12-48 months after a cardiac condition diagnosis.

\section{Author affiliations}

${ }^{1}$ Leicester Diabetes Centre, University Hospitals of Leicester NHS Trust, Leicester, UK
${ }^{2}$ Institute of Psychological Research, De Montfort University, Leicester, UK ${ }^{3}$ Diabetes Research Centre, College of Life Sciences, University of Leicester, Leicester, UK

${ }^{4}$ Department of Heath Sciences, College of Life Sciences, University of Leicester, Leicester, UK

${ }^{5}$ Glenfield Hospital, University Hospitals of Leicester NHS Trust, Leicester, UK

Acknowledgements The TEXTME Message Bank was developed and validated by The University of Sydney \& The George Institute and was used under licence. Finally, thank you to all the study participants, staff and facilitators, without whose hard work the study would not have been possible.

Contributors LYH contributed to design, development, planning, recruitment, conduct, analysis and reporting. HD contributed to design, development, planning, conduct, analysis and reporting. SS contributed to design, development, planning, recruitment, conduct and reporting. EJS contributed to recruitment and conduct. GW contributed to analysis and reporting. LJG contributed to design, development, analysis and reporting. KK contributed to design, development and reporting. TY contributed to design, development, analysis and reporting study. PJH contributed to analysis and reporting. AVR contributed to analysis and reporting. IH contributed to design and reporting. SS contributed to design and reporting. MJD contributed to design, development, planning, recruitment, conduct, analysis and reporting and is responsible for the overall content as guarantor.

Funding This study was funded by the NIHR Collaboration for Leadership in Applied Health Research and Care East Midlands (CLAHRC EM), now recommissioned as NIHR Applied Research Collaboration East Midlands (ARC EM).

Disclaimer The views expressed are those of the authors and not necessarily those of the NIHR, the Department of Health and Social Care or the NHS.

Competing interests MJD reports personal fees from Novo Nordisk, Sanofi, Eli Lilly, Boehringer Ingelheim, AstraZeneca, Janssen, Intarcia/Servier, Gilead Sciences, NAPP, Merck, Sharp and Dohme, Mitsubishi Tanabe Pharma Corporation, Takeda Pharmaceuticals International Inc and grants from AstraZeneca, Novo Nordisk, Boehringer Ingelheim, Janssen, Sanofi, outside the submitted work. TY reports grants from NIHR Leicester BRC, during the conduct of the study.

Patient consent for publication Not required.

Ethics approval The West Midlands-Solihull Research Ethics Committee and the UK Health Research Authority (Reference: 16/WM/0463) granted approval.

Provenance and peer review Not commissioned; externally peer reviewed.

Data availability statement Data are available on reasonable request. Individual participant data that underlie the results reported in this article, after deidentification (text, tables, figures and appendices) along with the study protocol and statistical analysis plan, will be available 12 months post publication on reasonable request to researchers who provide a methodologically sound proposal, to achieve the aims outlined in their proposal. Proposals should be directed to melanie.davies@uhl-tr.nhs.uk. To gain access, data requestors will need to sign a data sharing agreement.

Open access This is an open access article distributed in accordance with the Creative Commons Attribution Non Commercial (CC BY-NC 4.0) license, which permits others to distribute, remix, adapt, build upon this work non-commercially, and license their derivative works on different terms, provided the original work is properly cited, appropriate credit is given, any changes made indicated, and the use is non-commercial. See: http://creativecommons.org/licenses/by-nc/4.0/.

Correction notice This article has been corrected since it first published. The provenance and peer review statement has been included.

\section{ORCID iDs}

Louisa Y Herring http://orcid.org/0000-0002-9592-6759

Helen Dallosso http://orcid.org/0000-0002-6732-0864

Samuel Seidu http://orcid.org/0000-0002-8335-7018

\section{REFERENCES}

1 Darden D, Richardson C, Jackson EA. Physical activity and exercise for secondary prevention among patients with cardiovascular disease. Curr Cardiovasc Risk Rep 2013;7:411-6.

2 Varghese T, Schultz WM, McCue AA, et al. Physical activity in the prevention of coronary heart disease: implications for the clinician. Heart 2016;102:904-9. 
3 Anderson L, Thompson DR, Oldridge N, et al. Exercise-based cardiac rehabilitation for coronary heart disease. Cochrane Database Syst Rev 2016:CD001800.

4 British Heart Foundation. The national audit of cardiac rehabilitation (NACR) Quality and outcomes Report 2018, 2018.

5 Fell J, Dale V, Doherty P. Does the timing of cardiac rehabilitation impact fitness outcomes? An observational analysis. Open Heart 2016;3:e000369.

6 ter Hoeve N, Huisstede BMA, Stam HJ, et al. Does cardiac rehabilitation after an acute cardiac syndrome lead to changes in physical activity habits? systematic review. Phys Ther 2015:95:167-79.

7 Herring LY, Dallosso H, Chatterjee S, et al. Physical Activity after Cardiac EventS (PACES) - a group education programme with subsequent text-message support designed to increase physical activity in individuals with diagnosed coronary heart disease: study protocol for a randomised controlled trial. Trials 2018;19:537.

8 Davies MJ, Gray LJ, Troughton J, et al. A community based primary prevention programme for type 2 diabetes integrating identification and lifestyle intervention for prevention: the Let's Prevent Diabetes cluster randomised controlled trial. Prev Med 2016;84:48-56.

9 Chow CK, Redfern J, Hillis GS, et al. Effect of Lifestyle-Focused text messaging on risk factor modification in patients with coronary heart disease: a randomized clinical trial. JAMA 2015;314:1255-63.

10 Park LG, Howie-Esquivel J, Chung ML, et al. A text messaging intervention to promote medication adherence for patients with coronary heart disease: a randomized controlled trial. Patient Educ Couns 2014:94:261-8.

$11 \mathrm{Kim} \mathrm{BH}$, Glanz K. Text messaging to motivate walking in older African Americans: a randomized controlled trial. Am J Prev Med 2013;44:71-5.

12 Singh SJ, Jones PW, Evans R, et al. Minimum clinically important improvement for the incremental shuttle walking test. Thorax 2008:63:775-7.

13 British Heart Foundation. Put your heart into walking. London: British heart Foundation (BHF), 2014

14 Redfern J, Thiagalingam A, Jan S, et al. Development of a set of mobile phone text messages designed for prevention of recurrent cardiovascular events. Eur J Prev Cardiol 2014;21:492-9.

15 Golubic R, May AM, Benjaminsen Borch K, et al. Validity of electronically administered recent physical activity questionnaire (RPAQ) in ten European countries. PLoS One 2014:9:e92829.

16 Herrmann C. International experiences with the Hospital Anxiety and Depression Scale--a review of validation data and clinical results. $J$ Psychosom Res 1997;42:17-41.

17 EuroQol Research Foundation. How to use EQ-5D, 2016. Available: http://www.euroqol.org/about-eq-5d/how-to-use-eq-5d.html
18 Resnick B, Jenkins LS. Testing the reliability and validity of the selfefficacy for exercise scale. Nurs Res 2000;49:154-9.

19 Valenti L, Lim L, Heller RF, et al. An improved questionnaire for assessing quality of life after acute myocardial infarction. Qual Life Res 1996;5:151-61.

20 Bell JA, Hamer M, van Hees VT, et al. Healthy obesity and objective physical activity. Am J Clin Nutr 2015;102:268-75.

21 Migueles JH, Rowlands AV, Huber F, et al. GGIR: a research Community-Driven open source $\mathrm{R}$ package for generating physical activity and sleep outcomes from Multi-Day RAW Accelerometer data. J Meas Phys Behav 2019;2:188-96.

22 Hildebrand M, VAN Hees VT, Hansen BH, et al. Age group comparability of raw accelerometer output from wrist- and hip-worn monitors. Med Sci Sports Exerc 2014;46:1816-24.

23 Rubin DB. Inference and missing data. Biometrika 1976;63:581-92.

24 Dibben GO, Dalal HM, Taylor RS, et al. Cardiac rehabilitation and physical activity: systematic review and meta-analysis. Heart 2018;104:1394-402.

25 de Souto Barreto P, Ferrandez A-M, Saliba-Serre B. Are older adults who volunteer to participate in an exercise study fitter and healthier than nonvolunteers? the participation bias of the study population. $J$ Phys Act Health 2013;10:359-67.

26 Hur SA, Guler SA, Khalil N, et al. Minimal important difference for physical activity and validity of the International physical activity questionnaire in interstitial lung disease. Ann Am Thorac Soc 2019;16:107-15.

27 Campo G, Tonet E, Chiaranda G, et al. Exercise intervention improves quality of life in older adults after myocardial infarction: randomised clinical trial. Heart 2020;106:1658-64.

28 Shepherd CW, While AE. Cardiac rehabilitation and quality of life: a systematic review. Int J Nurs Stud 2012;49:755-71.

29 de Silva D. Helping measure person-centred care: a review of evidence about commonly used approaches and tools used to help measure person-centred care. The health Foundation Inspiring improvement, 2014. Available: https://www.health.org.uk/ publications/helping-measure-person-centred-care

30 Winkley K, Stahl D, Amiel SA, et al. A multi-level model to explain attendance at structured diabetes education for people with newly diagnosed type 2 diabetes. Eur J Public Health 2014;24.

31 NHS Digital. National diabetes audit report 1 Care processes and treatment targets 2017-18. UK, 2018.

32 Yates T, Haffner SM, Schulte PJ, et al. Association between change in daily ambulatory activity and cardiovascular events in people with impaired glucose tolerance (navigator trial): a cohort analysis. Lancet 2014;383:1059-66. 
Correction: Physical Activity after Cardiac EventS (PACES): a group education programme with subsequent text message support designed to increase physical activity in individuals with diagnosed coronary heart disease: a randomised controlled trial

Herring LY, Dallosso H, Schreder S, et al. Physical Activity after Cardiac EventS (PACES): a group education programme with subsequent text message support designed to increase physical activity in individuals with diagnosed coronary heart disease: a randomised controlled trial. Open Heart 2021;8:e001351. doi:10.1136/openhrt2020-001351

This article has been corrected since it was first published. The provenance and peer review statement has been included.

Open access This is an open access article distributed in accordance with the Creative Commons Attribution Non Commercial (CC BY-NC 4.0) license, which permits others to distribute, remix, adapt, build upon this work non-commercially, and license their derivative works on different terms, provided the original work is properly cited, appropriate credit is given, any changes made indicated, and the use is non-commercial. See: http://creativecommons.org/licenses/by-nc/4.0/.

C Author(s) (or their employer(s)) 2021. Re-use permitted under CC BY-NC. No commercial re-use. See rights and permissions. Published by BMJ.

Open Heart 2021;8:e001351corr1. doi:10.1136/openhrt-2020-001351corr1

(A) Check for updates 